\title{
CONVOLUTION EQUATIONS IN CERTAIN BANACH SPACES
}

\author{
ALEXANDER L. KOLDOBSKII
}

(Communicated by William J. Davis)

\begin{abstract}
For a Banach space $E$ and $p>0$, the following problem is considered: how to identify a finite Borel measure $\mu$ on $E$ by means of the potential $g(a)=\int_{E}\|x-a\|^{p} d \mu(x), a \in E$. The solution for infinite-dimensional Hilbert spaces is based on limit correlations between the Fourier transforms of finite-dimensional restrictions of $g$ and $\|x\|^{p}$. For finite-dimensional subspaces of $L_{p}$, the Levy representation of norms is used.
\end{abstract}

\section{INTRODUCTION}

Let $(E,\|\cdot\|)$ be a Banach space, $M_{p}$ be the set of Borel measures $\mu$ on $E$ satisfying $\int_{E}(1+\|x\|)^{p} d \mu(x)<\infty$. For $\mu \in M_{p}$, let $g$ be the potential of $\mu$ :

$$
g(a)=\int_{E}\|x-a\|^{p} d \mu(x), a \in E .
$$

The problem is to identify $\mu$ by means of $g$.

Thus we are going to study a sort of convolution equation. The uniqueness problem for this equation has been investigated by several authors. For some special Banach spaces $E$ the solution of this equation is unique for all $p>0$, except for a countable set of exponents $p$, which will be called exceptional for the space $E$. For instance, in the one-dimensional case the exceptional exponents are the even numbers, [19-21], and the same is true for separable Hilbert spaces [see [22, 1, 9] for the finite-dimensional case and [10], [15] for the infinite-dimensional case]. Exceptional for $L_{q}$-spaces are the numbers $p$, for which $p / q \in \mathbb{N}$ and, besides that, in the case of $n$-dimensional space $l_{q}^{n}$ one of the following three conditions must be fulfilled: (a) $p / q<n$; (b) $q$ is an even integer; (c) $q$ and $(p / q)-n$ are odd integers. For the complex space $l_{\infty}^{n}$, even integers are exceptional; and for the real space, $p$ is exceptional iff $n+p$ is odd. The set of exceptional exponents $p>0$ is empty for spaces $C(K)$,

Received by the editors May 22, 1989 and, in revised form, January 25, 1990.

1980 Mathematics Subject Classification (1985 Revision). Primary 46F12, 45E10; Secondary 46G12, 60B11. 
where $K$ is an infinite metric compact space without isolated points, and for spaces $C_{0}(\Omega)$, where $\Omega$ is a noncompact, locally compact Hausdorff space. If $K$ contains isolated points, then positive $p \notin \mathbb{N}$ are not exceptional, and the problem is still open for $p \in \mathbb{N}$ [for all these results, see $[5,6,11,16$ ].

If $E$ is a finite-dimensional space, the potential $g$ can be considered as the convolution of distributions $\|x\|^{p}$ and $\mu$ over the space $S=S\left(\mathbb{R}^{n}\right)$ of rapidly decreasing functions. This convolution is, as a rule, naturally connected with the Fourier transform [6], so to solve the convolution equation one can compute $\left(\|x\|^{p}\right)^{\wedge}$, verify that $\left(\|x\|^{p}\right)^{\wedge} \neq 0$ on open sets and put $\hat{\mu}=\hat{g} /\left(\|x\|^{p}\right)^{\wedge}$ (throughout we denote by $\hat{f}$ the Fourier transform of a distribution $f$ ). However, the complete solution of the inverse problem has been obtained only in the case $E=\mathbb{R}^{n}$, when $\left(\|x\|^{p}\right)^{\text {一 }}$ can easily be computed. For other finitedimensional spaces only uniqueness theorems are available, because attempts at straightforward computation of $\left(\|x\|^{p}\right)^{\wedge}$ have been unsuccessful. In the following, an analytic continuation of $\|x\|^{p}$ was used to check that $\left(\|x\|^{p}\right)^{\wedge} \neq 0$ on open sets.

In this paper the Fourier transform of norms in some finite-dimensional spaces is computed with the help of isometric embedding of these spaces into $L_{p}$. In $\S 4$ this is done for an arbitrary $n$-dimensional subspace $E=$ $\operatorname{span}\left(f_{1}, \ldots, f_{n}\right)$ of $L_{p}$. If $p \neq 2,4,6, \ldots$ then $p$ is exceptional for $E$ iff the joint distribution of $f_{1}, \ldots, f_{n}$ vanishes on some open cone in $\mathbb{R}^{n}$. (We recall that this joint distribution is a measure on $\mathbb{R}^{n}$.) In the same section, any easy proof is obtained for the well-known equimeasurability theorem for $L_{p}$-isometries [19-21, 17, 7, 12].

In $\S 5$ we give some concrete consequences of results of $\S 4$. The construction of isometric embedding of $l_{q}^{n}$ into $L_{p}(0<p<q<2)$ from [2] is used to obtain the Levy representation of norms and, as a consequence, to express the Fourier transform of $l_{q}^{n}$-norm in terms of $q$-stable measures. In $\S 6$ we investigate the inverse problem for two-dimensional spaces. An exponent $p>0$ is exceptional for $E=\operatorname{span}\left(e_{1}, e_{2}\right)$ iff the $(p+1)$ th fractional derivative of the function $\left\|e_{1}+t e_{2}\right\|^{p}$ vanishes on some open subset of $\mathbb{R}$. In particular, the exponent $p=1$ is exceptional for a two-dimensional space iff this space is not strictly convex.

A connection between the Fourier transform and isometric embedding into $L_{p}$ is discussed in $\S 3$. In particular, it is proved in this section that, for an arbitrary even function $h$ on $\mathbb{R}$ satisfying some mild additional assumptions, if $\int_{S^{n-1}} h(\langle x, \xi\rangle) d \nu(\xi)=0$ for each $x \in \mathbb{R}^{n}$, then either $\nu=0$ or $h$ is a polynomial containing even powers only (here $\langle x, \xi\rangle$ stands for the scalar product in $\mathbb{R}^{n}$ and $\nu$ is a symmetric charge on the unit sphere $S^{n-1}$ in $\mathbb{R}^{n}$ ). This statement for $h(t)=|t|^{p}$ has been proved by different methods in $[8,14$, 18 .

In $\S 2$ the convolution equation is solved for infinite-dimensional Hilbert spaces. This solution is based on limit correlations between the Fourier transforms of functions $g$ and $\|x\|^{p}$. 
All definitions and facts about distributions over $S\left(\mathbb{R}^{n}\right)$ used in this paper can be found in [3].

\section{Infinite-dimensional Hilbert spaces}

Let $E$ be the separable Hilbert space $l_{2}$ and $e_{i}, i \in \mathbb{N}$, be the standard basis in $l_{2}, E_{n}=\operatorname{span}\left(e_{i}, i=1, \ldots, n\right)$. For $x=\sum_{1}^{\infty} x_{i} e_{i} \in l_{2}$, we write $x^{(n)}=\sum_{1}^{n} x_{i} e_{i}$ (so $x^{(n)}$ is the projection of $x$ to $E_{n}$ ).

Let $\mu$ be a Borel measure on $l_{2}, p \in \mathbb{R}$. We shall assume that $\mu \in M_{p}$ if $p>0$, and that $\mu\left(l_{2}\right)<\infty$ if $p<0$. Note that the restriction $g_{n}$ of the potential $g(a)=\int_{E}\|x-a\|^{p} d \mu(x)$ to the subspace $E_{n}$ is locally an $L_{1^{-}}$ function on $E_{n}$ with respect to Lebesgue measure on $E_{n}$, if $-n<p<0$ (see, for instance, $\left[13\right.$, p. 84]). So $g_{n}$ can be considered as a distribution over $S\left(\mathbb{R}^{n}\right)$. Theorem 1. If $p \in \mathbb{R}, p \neq 0,2,4, \ldots$, then for each $\xi \in l_{2}, \xi \neq 0$,

$$
\hat{\mu}(\xi)=\lim _{n \rightarrow \infty} \frac{\hat{g}_{n}\left(\xi^{(n)}\right)\left\|\xi^{(n)}\right\|^{n+p} \Gamma(-p / 2)}{2^{n+p} \pi^{n / 2} \Gamma((n+p) / 2)}
$$

Proof. Let us fix an element $x \in l_{2}$ and assume first that $-1<p<0$. In order to compute the Fourier transform of the function $\|x-a\|^{p}$ of the variable $a \in E_{n}$, we can use the following representation, which is an easy consequence of the definition of the $\Gamma$-function:

$$
\|x-a\|^{p}=\frac{2}{\Gamma(-p / 2)} \int_{0}^{\infty} t^{-1-p} \exp \left(-t^{2}\|x-a\|^{2}\right) d t .
$$

Now for each fixed $t>0$, the Fourier transform of the function $a \mapsto$ $\exp \left(-t^{2}\|x-a\|^{2}\right), a \in E_{n}$, can easily be computed:

$\left(\exp \left(-t^{2}\|x-a\|^{2}\right)\right)^{\wedge}(\zeta)=\pi^{n / 2} t^{-n} \exp \left(-i\left\langle x^{(n)}, \zeta\right\rangle-t^{2}\left\|x-x^{(n)}\right\|^{2}-\|\zeta\|^{2} / 4 t^{2}\right)$, $\zeta \in \mathbb{R}^{n}$. Consequently, for every $\zeta \in \mathbb{R}^{n}, \zeta \neq 0$,

(1)

$$
\begin{aligned}
& \left(\|x-a\|^{p}\right)^{-}(\zeta) \\
& \quad=\frac{2 \pi^{n / 2}}{\Gamma(-p / 2)} \exp \left(-i\left\langle x^{(n)}, \zeta\right\rangle\right) \int_{0}^{\infty} t^{-1-p-n} \exp \left(-t^{2}\left\|x-x^{(n)}\right\|^{2}-\|\zeta\|^{2} / 4 t^{2}\right) d t .
\end{aligned}
$$

If we allow $p$ to assume complex values, then both sides of (1) are analytic functions of $p$ in the domain $\{\operatorname{Re} p>-n, p \neq 0,2,4,6, \ldots\}$. So these functions admit a unique analytic continuation from the interval $(-1,0)$, and (1) remains true for all real $p>-n, p \neq 0,2,4, \ldots$.

Making in (1) the change of variables $y=1 / t$ and then integrating against $d \mu(x)$, we obtain

(2)

$$
\begin{aligned}
\hat{g}_{n}(\zeta)= & \frac{2 \pi^{n / 2}}{\Gamma(-p / 2)} \times \int_{l_{2}} \exp \left(-i\left\langle x^{(n)}, \zeta\right\rangle\right) \\
& \times\left(\int_{0}^{\infty} y^{n+p-1} \exp \left(-y^{2}\|\zeta\|^{2} / 4-\left\|x-x^{(n)}\right\|^{2} / y^{2}\right) d y\right) d \mu(x)
\end{aligned}
$$


for every $\zeta \in \mathbb{R}^{n}, \zeta \neq 0$, and $p>-n, p \neq 0,2,4, \ldots$.

Now let us consider an arbitrary $p \in \mathbb{R}, p \neq 0,2,4, \ldots$, and $\xi \in l_{2}, \xi \neq 0$. For each $n \in \mathbb{N}$ with $\xi^{(n)} \neq 0$ and $p>-n$, put $\zeta=\xi^{(n)}$ in (2) and divide both sides of (2) by

$$
\frac{2 \pi^{n / 2}}{\Gamma(-p / 2)} \int_{0}^{\infty} y^{n+p-1} \exp \left(-y^{2}\left\|\xi^{(n)}\right\|^{2} / 4\right) d y=\frac{2^{n+p} \pi^{n / 2}\left\|\xi^{(n)}\right\|^{-n-p} \Gamma\left(\frac{n+p}{2}\right)}{\Gamma(-p / 2)}
$$

This leads to

$$
\begin{aligned}
\frac{\hat{g}_{n}\left(\xi^{(n)}\right)\left\|\xi^{(n)}\right\|^{n+p} \Gamma(-p / 2)}{2^{n+p} \pi^{n / 2} \Gamma\left(\frac{n+p}{2}\right)}=\int_{l_{2}} \exp \left(-i\left\langle x^{(n)}, \xi^{(n)}\right\rangle\right) \\
\quad \times \frac{\int_{0}^{\infty} y^{n+p-1} \exp \left(-y^{2}\left\|\xi^{(n)}\right\|^{2} / 4-\left\|x-x^{(n)}\right\|^{2} / y^{2}\right) d y}{\int_{0}^{\infty} y^{n+p-1} \exp \left(-y^{2}\left\|\xi^{(n)}\right\|^{2} / 4\right) d y} d \mu(x) .
\end{aligned}
$$

The absolute value of the integrand on the right is majorated by 1 and for each $x \in l_{2}$ tends to $\exp (-i\langle x, \xi\rangle)$ as $n \rightarrow \infty$. In fact, $1-\exp (-z)<z$ for $z>0$, so the difference between the fraction on the right-hand side of (3) and the number 1 is less than

$$
\left\|x-x^{(n)}\right\|^{2} \frac{\int_{0}^{\infty} y^{n+p-3} \exp \left(-y^{2}\left\|\xi^{(n)}\right\|^{2} / 4\right) d y}{\int_{0}^{\infty} y^{n+p-1} \exp \left(-y^{2}\left\|\xi^{(n)}\right\|^{2} / 4\right) d y}=\frac{\left\|x-x^{(n)}\right\|^{2}\left\|\xi^{(n)}\right\|^{2}}{2(n+p-2)},
$$

which tends to zero as $n \rightarrow \infty$. Now we can apply the Lebesgue dominated convergence theorem to obtain that the quantity on the right in (3) tends to $\hat{\mu}(\xi)$ as $n \rightarrow \infty$.

\section{UNIQUENESS THEOREM FOR MEASURES ON $S^{n-1}$}

Let $H$ denote the set of even, continuous functions $h$ on $\mathbb{R}$ with tempered growth at infinity (i.e., $\lim _{|x| \rightarrow \infty}\left(h(x) /|x|^{\rho}\right)=0$ for some $\rho>0$ ), for which $\hat{h}$ is a continuous function on $\mathbb{R} \backslash\{0\}$ with tempered growth at infinity.

Let $\nu$ be a symmetric Borel charge of bounded variation on the unit sphere $S^{n-1}$ in $\mathbb{R}^{n}$.

The distribution $\hat{h}(t) d \nu(\xi)$ is defined by

$$
\langle\hat{h}(t) d \nu(\xi), \varphi\rangle=\int_{S^{n-1}} d \nu(\xi) \int_{\mathbb{R}} \hat{h}(t) \varphi(t \xi) d t=\int_{S^{n-1}}\langle\hat{h}, \varphi(t \xi)\rangle d \nu(\xi)
$$

for each $\varphi \in S\left(\mathbb{R}^{n}\right)$ with $0 \notin \operatorname{supp} \varphi$.

Lemma 1. For every $\xi_{0} \in S^{n-1}$, the Fourier transform of the function $h\left(\left\langle x, \xi_{0}\right\rangle\right)$ coincides on $\mathbb{R}^{n} \backslash\{0\}$ with the distribution $\hat{h}(t) d \delta_{\xi_{0}}(\xi)$, where $\delta_{\xi_{0}}$ is the unit mass at the point $\xi_{0}$. 
Proof. By the Fubini theorem, for every even function $\varphi \in S\left(\mathbb{R}^{n}\right)$ with $0 \notin$ $\operatorname{supp} \varphi$, we have

$$
\begin{aligned}
\left\langle\left(h\left(\left\langle x, \xi_{0}\right\rangle\right)\right)^{-}, \varphi\right\rangle & =\left\langle h\left(\left\langle x, \xi_{0}\right\rangle\right), \hat{\varphi}\right\rangle \\
& =\int_{\mathbb{R}^{n}} h\left(\left\langle x, \xi_{0}\right\rangle\right) \hat{\varphi}(x) d x=\int_{\mathbb{R}} h(t)\left(\int_{\left\langle x, \xi_{0}\right\rangle=t} \hat{\varphi}(x) d x\right) d t .
\end{aligned}
$$

The even function $\varphi\left(t \xi_{0}\right)$ is the Fourier transform of the even function $\int_{\left\langle x, \xi_{0}\right\rangle=t} \hat{\varphi}(x) d x$ of the variable $t \in \mathbb{R}$. (It is a simple property of a Radon transform $[4$, p. 19].) So we have

$$
\left\langle\left(h\left(\left\langle x, \xi_{0}\right\rangle\right)\right)^{-}, \varphi\right\rangle=\left\langle h, \int_{\left\langle x, \xi_{0}\right\rangle=t} \hat{\varphi}(x) d x\right\rangle=\left\langle\hat{h}, \varphi\left(t \xi_{0}\right)\right\rangle=\left\langle\hat{h}(t) d \delta_{\xi_{0}}(\xi), \varphi\right\rangle
$$

and even distributions $\left(h\left(\left\langle x, \xi_{0}\right\rangle\right)\right)^{\wedge}$ and $\hat{h}(t) d \delta_{\xi_{0}}(\xi)$ coincide on $\mathbb{R}^{n} \backslash\{0\}$.

The next result is a consequence of Lemma 1 and the Fubini theorem:

Lemma 2. Let $h \in H$ and $\nu$ be a symmetric Borel charge of bounded variation on $S^{n-1}$. Then the Fourier transform of the function

$$
f(x)=\int_{S^{n-1}} h(\langle x, \xi\rangle) d \nu(\xi)
$$

coincides on $\mathbb{R}^{n} \backslash\{0\}$ with the distribution $\hat{h}(t) d \nu(\xi)$.

Proof. For an arbitrary even function $\varphi \in S\left(\mathbb{R}^{n}\right)$ with $0 \notin \operatorname{supp} \varphi$

$$
\begin{aligned}
\langle\hat{f}, \varphi\rangle & =\langle f, \hat{\varphi}\rangle=\int_{S^{n-1}} d \nu(\xi) \int_{\mathbb{R}^{n}} h(\langle x, \xi\rangle) \hat{\varphi}(x) d x \\
& =\int_{S^{n-1}} d \nu(\xi) \int_{\mathbb{R}} \hat{h}(t) \varphi(t \xi) d t=\langle\hat{h}(t) d \nu(\xi), \varphi\rangle .
\end{aligned}
$$

Theorem 2. If $\int_{S^{n-1}} h(\langle x, \xi\rangle) d \nu(\xi)=0$ for all $x \in \mathbb{R}^{n}$, then either $\nu=0$ or $h$ is a polynomial containing even powers only.

Proof. By Lemma 2, $\hat{h}(t) d \nu(\xi)=0$ everywhere on $\mathbb{R}^{n} \backslash\{0\}$. This is possible only if either $\nu=0$ or $\hat{h}$ is a distribution with support in $\{0\}$. Since $h$ is even, in the last case $h$ must be a polynomial containing even powers only.

\section{Finite-dimensional subsPaCes of $L_{p}$}

Let $(E,\|\cdot\|)$ be an $n$-dimensional subspace of $L_{p}(\Omega, \sigma)$, where $p>0$ and $(\Omega, \sigma)$ is a measure space, $\sigma(\Omega)<\infty$.

Consider an arbitrary basis $f_{1}, \ldots, f_{n}$ in $E$. Let $\mu$ be the joint distribution of functions $f_{1}, \ldots, f_{n}$ with respect to $\sigma$; that is, $\mu(B)=$ $\sigma\left\{\omega \in \Omega:\left(f_{1}(\omega), \ldots, f_{n}(\omega)\right) \in B\right\}$ for every Borel subset $B$ of $\mathbb{R}^{n}$. Note that $\mu$ is a finite Borel measure on $\mathbb{R}^{n}$. 
A measure $\nu$ on the unit sphere $S^{n-1}$ in $\mathbb{R}^{n}$, defined by

$$
\nu(B)=\frac{1}{2} \int_{B \times \mathbb{R}}\|x\|_{2}^{p} d \mu(x)
$$

for all Borel subsets $B$ of $S^{n-1}$, will be called the $p$-projection of $\mu$ to $S^{n-1}$. (Here $\|x\|_{2}$ is the Euclidean norm, $B \times \mathbb{R}=\left\{y \in \mathbb{R}^{n}, y \neq 0: y /\|y\|_{2} \in B \cup\right.$ $(-B)\}$.

The norm in $E$ can be represented in the following way:

$$
\begin{aligned}
\|x\|^{p} & =\left\|\sum_{1}^{n} x_{i} f_{i}\right\|^{p}=\int_{\Omega}\left|\sum_{1}^{n} x_{i} f_{i}(\omega)\right|^{p} d \sigma(\omega)=\int_{\mathbb{R}^{n}}|\langle x, \xi\rangle|^{p} d \mu(\xi) \\
& =\int_{S^{n-1}}|\langle x, \xi\rangle|^{p} d \nu(\xi) .
\end{aligned}
$$

(In such situations we shall say that the norm in $E$ admits the Levy representation with the measure $\mu$.)

Now we apply Lemma 2 with $h(y)=|y|^{p}, y \in \mathbb{R}$, to compute the Fourier transform of $\|x\|^{p}$. Note $\left(|y|^{p}\right)^{\wedge}(t)=\left(2^{p+1} \sqrt{\pi} \Gamma((p+1) / 2) / \Gamma(-p / 2)\right)|t|^{-1-p}$, if $p>0, p \neq 2,4,6, \ldots[3$, p. 217].

Lemma 3. If $p>0, p \neq 2,4,6, \ldots$ then

$$
\left(\|x\|^{p}\right)^{\wedge}=\frac{2^{p+1} \sqrt{\pi} \Gamma((p+1) / 2)}{\Gamma(-p / 2)}|t|^{-1-p} d \nu(\xi) .
$$

It is clear now that $\left(\|x\|^{p}\right)^{\wedge}=0$ on an open subset of $\mathbb{R}^{n}$ iff $\nu=0$ on some open subset of $\mathbb{R}^{n-1}$. So we have the following:

Theorem 3. If $p>0, p \neq 2,4,6, \ldots$ then $p$ is an exceptional exponent for the space $E=\operatorname{span}\left(f_{1}, \ldots, f_{n}\right) \subset L_{p}(\Omega, \sigma)$ iff $\mu(B \times \mathbb{R})=0$ for some open subset $B$ of $S^{n-1}$, where $\mu$ is the joint distribution of functions $f_{1}, \ldots, f_{n}$ with respect to $\sigma$.

Suppose that $\Omega$ is a topological space, $\sigma$ is a finite Borel measure on $\Omega$ which does not vanish on open sets, and $f_{1}, \ldots, f_{n}$ are continuous functions on $\Omega$. Let $V$ be the subset of $\mathbb{R}^{n-1}$ consisting of all points of the form $\left(f_{2}(\omega) / f_{1}(\omega), \ldots, f_{n}(\omega) / f_{1}(\omega)\right)$ or $\left(-f_{2}(\omega) / f_{1}(\omega), \ldots,-f_{n}(\omega) / f_{1}(\omega)\right)$, where $\omega$ runs over the set $\Omega \backslash f_{1}^{-1}(0)$. In this case $\mu(B \times \mathbb{R}) \neq 0$ for all open subsets $B$ of $S^{n-1}$ iff $V$ is dense in $\mathbb{R}^{n-1}$.

Example. Let $\Omega=S^{1}$ be the unit circle in $\mathbb{R}^{2}$ with (linear) Lebesgue measure, $p>0, p \neq 2,4,6, \ldots$. Then for the space $E_{1}=\operatorname{span}(\sin \omega, \sin 2 \omega) \subset$ $L_{p}\left(S^{1}\right)$, we have $V=(-2,2)$, and $p$ is an exceptional exponent for $E_{1}$. If $E_{2}=\operatorname{span}(\sin 2 \omega, \sin 3 \omega) \subset L_{p}\left(S^{1}\right)$, then $V=\mathbb{R}$, and $p$ is not exceptional for $E_{2}$.

Let $(\Omega, \mathscr{B}, \sigma)$ and $\left(\Omega^{\prime}, \mathscr{B}^{\prime}, \sigma^{\prime}\right)$ be measure spaces with finite measures, $p>0, p \neq 2,4,6, \ldots$ and $Y$ be an arbitrary (maybe infinite-dimensional) subspace of $L_{p}(\Omega)$. Suppose that a linear isometry $T$ maps $Y$ into $L_{p}\left(\Omega^{\prime}\right)$. 
The well-known continuation theorem for $L_{p}$-isometries [see 19, 20, or 7] states that $T$ can be extended to the space $L_{p}\left(\Omega, \mathscr{B}_{0}, \sigma\right)$ as a linear isometry, where $\mathscr{B}_{0}$ is a minimal $\sigma$-algebra contained in $\mathscr{B}$, making all functions in the space $Y$ measurable.

This result was obtained as a straightforward consequence of the following equimeasurability theorem for $L_{p}$-isometries [see 19-21, 17, 7]:

Theorem 4. For arbitrary functions $f_{1}, \ldots, f_{n} \in Y$ we have $\mu_{1}=\mu_{2}$, where measures $\mu_{1}$ and $\mu_{2}$ on $\mathbb{R}^{n}$ are the joint distributions of the $n$-tuples

$$
\left(\mathbf{1}, f_{2}(\omega) / f_{1}(\omega), \ldots, f_{n}(\omega) / f_{1}(\omega)\right)
$$

and

$$
\left(\mathbf{1}, T f_{2}\left(\omega^{\prime}\right) / T f_{1}\left(\omega^{\prime}\right), \ldots, T f_{n}\left(\omega^{\prime}\right) / T f_{1}\left(\omega^{\prime}\right)\right)
$$

with respect to measures $\left|f_{1}\right|^{p} d \sigma$ and $\left|T f_{1}\right|^{p} d \sigma^{\prime}$ accordingly.

Lemma 2, above, provides a simple proof of Theorem 4. Indeed, $\left\|\sum_{1}^{n} x_{i} f_{i}\right\|^{p}$ $=\left\|\sum_{1}^{n} x_{i} T f_{i}\right\|^{p}$ for each $x=\left(x_{1}, \ldots, x_{n}\right) \in \mathbb{R}^{n}$, so

$$
\begin{aligned}
& \int_{\Omega}\left|\sum_{1}^{n} x_{i} f_{i}(\omega)\right|^{p} d \sigma(\omega) \\
& \quad=\int_{f_{1}(\omega) \neq 0}\left|x_{1}+\sum_{2}^{n} x_{i} \frac{f_{i}(\omega)}{f_{1}(\omega)}\right|^{p}\left|f_{1}(\omega)\right|^{p} d \sigma(\omega)+\int_{f_{1}(\omega)=0}\left|\sum_{2}^{n} x_{i} f_{i}(\omega)\right|^{p} d \sigma(\omega) \\
& \quad=\int_{\mathbb{R}^{n}}|\langle x, \xi\rangle|^{p} d \mu_{1}(\xi)+\psi\left(x_{2}, \ldots, x_{n}\right) \\
& =\int_{\Omega^{\prime}}\left|\sum_{1}^{n} x_{i} T f_{i}\left(\omega^{\prime}\right)\right|^{p} d \sigma^{\prime}\left(\omega^{\prime}\right)=\int_{\mathbb{R}^{n}}|\langle x, \xi\rangle|^{p} d \mu_{2}(\xi)+\psi^{\prime}\left(x_{2}, \ldots, x_{n}\right) .
\end{aligned}
$$

Consider the Fourier transforms of these functions of variables $x_{1}, \ldots, x_{n}$. The Fourier transforms of functions $\psi$ and $\psi^{\prime}$ are supported on the hyperplane $\xi_{1}=0$ in $\mathbb{R}^{n}$. The measures $\mu_{1}$ and $\mu_{2}$ are supported on the hyperplane $\xi_{1}=1$ in $\mathbb{R}^{n}$ so the $p$-projections $\nu_{1}$ and $\nu_{2}$ of these measures to $S^{n-1}$ are supported out of the hyperplane $\xi_{1}=0$. By Lemma 2, $\nu_{1}=\nu_{2}$, and therefore $\mu_{1}=\mu_{2}$.

\section{LEVY REPRESENTATIONS}

Let $(E,\|\cdot\|)$ be an $n$-dimensional Banach space. Suppose that there exists an even function $f \in L_{1}(\mathbb{R})$ with $(f(\|x\|))^{\wedge}=u \in L_{1}\left(\mathbb{R}^{n}\right)$. Then for every $k \in \mathbb{R}$ and $x \in E, x \neq 0$

$$
(2 \pi)^{n} f(k\|x\|)=\int_{\mathbb{R}^{n}} \exp (i k\|x\|(\langle x, \xi\rangle /\|x\|)) u(\xi) d \xi=\int_{\mathbb{R}} \exp (i k\|x\| y) d u_{1}(y),
$$

where $u_{1}$ is the image of the charge $u(\xi) d \xi$ under the mapping $\xi \mapsto\langle x, \xi\rangle /$ $\|x\|$. Here $k$ is arbitrary; therefore, $u_{1}=\hat{f}$, and $u_{1}$ does not depend on $x \in E, x \neq 0$. 
Now for every $p \in \mathbb{R}$ we obtain, assuming that the first integral converges:

$$
\int_{\mathbb{R}^{n}}|\langle x, \xi\rangle|^{p} u(\xi) d \xi=\|x\|^{p} \int_{\mathbb{R}^{n}}\left|\frac{\langle x, \xi\rangle}{\|x\|}\right|^{p} u(\xi) d \xi=\|x\|^{p} \int_{\mathbb{R}}|t|^{p} d u_{1}(t) .
$$

So if the $p$ th moment of the charge $u_{1}$ exists and is not zero then we have the Levy representation with the charge $u(\xi) d \xi$ :

$$
\|x\|^{p}=\frac{1}{\int_{\mathbb{R}}|t|^{p} d u_{1}(t)} \int_{\mathbb{R}^{n}}|\langle x, \xi\rangle|^{p} u(\xi) d \xi .
$$

In order to compute the moment, we assume that $p \in(-1,0)$ and use the Parseval theorem:

$$
\frac{2^{p+1} \sqrt{\pi} \Gamma((p+1) / 2)}{\Gamma(-p / 2)} \int_{\mathbb{R}}|t|^{-1-p} f(t) d t=\int_{\mathbb{R}}|t|^{p} d u_{1}(t) .
$$

If both sides of (5) are analytic functions of the variable $p$ in some domain in $\mathbb{C}$, we can use analytic continuation and compute moments for other $p$ 's.

For instance, if $E=l_{q}^{n}, f(t)=\exp \left(-|t|^{q}\right)$ and $0<p<q \leq 2$, then $u(\xi)=\gamma_{q}(\xi)=\left(\exp \left(-\|x\|_{q}^{q}\right)\right) \wedge(\xi)$ is the density of the $n$-dimensional $q$-stable measure, and $u_{1}$ is the one-dimensional stable measure. The $p$ th moment of $u_{1}$ is finite and can easily be computed [23, p. 75]. Indeed, for $p \in(-1,0)$

$$
\int_{\mathbb{R}}|t|^{-1-p} \exp \left(-|t|^{q}\right) d t=\frac{2 \Gamma(-p / q)}{q},
$$

and it follows from (5) and the analytic continuation argument that, for every $p \in(0, q)$,

$$
\int_{\mathbb{R}}|t|^{p} d u_{1}(t)=\frac{\Gamma(-p / q) 2^{p+2} \sqrt{\pi} \Gamma((p+1) / 2)}{q \Gamma(-p / 2)} .
$$

Now use (4) to get the Levy representation

$$
\|x\|_{q}^{p}=\frac{q \Gamma(-p / 2)}{2^{p+2} \sqrt{\pi} \Gamma((p+1) / 2) \Gamma(-p / q)} \int_{\mathbb{R}^{n}}|\langle x, \xi\rangle|^{p} d \gamma_{q}(\xi) .
$$

Now we can use Lemma 2 to compute the Fourier transform of the function $\|x\|_{q}^{p}$. Let $\nu_{p, q}$ be the $p$-projection of the $q$-stable measure $\gamma_{q}(\xi) d \xi$ onto $S^{n-1}$

Theorem 5. If $0<p<q<2$ then the Fourier transform of the function $\|x\|_{q}^{p}$ coincides on $\mathbb{R}^{n} \backslash\{0\}$ with the distribution $(q /(2 \Gamma(-p / q)))|t|^{-1-p} d \nu_{p, q}(\xi)$. (For the definition of this distribution, see $\S 3$.)

\section{TWO-DIMENSIONAL SPACES}

In this section we shall investigate the inverse problem for two-dimensional spaces.

Let $\mathscr{D}(\Omega)$ denote the space of functions from $S\left(\mathbb{R}^{2}\right)$ with compact support in an open set $\Omega \subset \mathbb{R}^{2}$, and $G=\mathbb{R}^{2} \backslash\{(x, y): y=0\}$. 
We shall say that a two-dimensional Banach space $E=\operatorname{span}\left(e_{1}, e_{2}\right)$ admits the Levy representation with an exponent $p>0, p \neq 2,4,6, \ldots$ and a distribution $\gamma$ over $S(\mathbb{R})$ if, for every function $\varphi \in S\left(\mathbb{R}^{2}\right)$ with $\hat{\varphi} \in \mathscr{D}(G)$, the following equality holds:

$$
\int_{\mathbb{R}^{2}}\left\|x e_{1}+y e_{2}\right\|^{p} \varphi(x, y) d x d y=\left\langle\gamma(\xi), \int_{\mathbb{R}^{2}}|x \xi-y|^{p} \varphi(x, y) d x d y\right\rangle .
$$

If $\hat{\varphi} \in \mathscr{D}(G)$, then it follows from Lemma 1 that

$$
\psi(\xi)=\int_{\mathbb{R}^{2}}|x \xi-y|^{p} \varphi(x, y) d x d y=\frac{C_{p}}{(2 \pi)^{2}} \int_{\mathbb{R}}|t|^{-1-p} \hat{\varphi}(t \xi,-t) d t,
$$

and the function $\psi$ belongs to $\mathscr{D}(\mathbb{R}) .\left(C_{p}=2^{p+1} \sqrt{\pi} \Gamma((p+1) / 2) / \Gamma(-p / 2)\right.$, here.) Moreover, for every function $\psi \in \mathscr{D}(\mathbb{R})$, there exists an even function $\varphi \in S\left(\mathbb{R}^{2}\right)$ with $\hat{\varphi} \in \mathscr{D}(G)$ for which (8) holds. Therefore, the distribution $\gamma$ in (7) is unique, if it exists.

If the space admits a Levy representation, then the Fourier transform of the norm can be computed. Indeed, for each function $\varphi \in \mathscr{D}(G)$, we get from (7) and (8) that

$$
\begin{aligned}
\left\langle\left(\left\|x e_{1}+y e_{2}\right\|^{p}\right)^{-}, \varphi\right\rangle & =\int_{\mathbb{R}^{2}}\left\|x e_{1}+y e_{2}\right\|^{p} \hat{\varphi}(x, y) d x d y \\
& =\left\langle\gamma(\xi), \int_{\mathbb{R}^{2}}|x \xi-y|^{p} \hat{\varphi}(x, y) d x d y\right\rangle \\
& =C_{p}\left\langle\gamma(\xi), \int_{\mathbb{R}}|t|^{-1-p} \varphi(t \xi,-t) d t\right\rangle .
\end{aligned}
$$

Lemma 4. $\left(\left\|x e_{1}+y e_{2}\right\|^{p}\right)^{\wedge}=0$ on some open set in $\mathbb{R}^{2}$ iff $\gamma=0$ on some open set in $\mathbb{R}$.

Now we shall prove the existence of a Levy representation for every twodimensional space and get a suitable expression for $\gamma$.

For an arbitrary $\varphi \in S\left(\mathbb{R}^{2}\right)$ let us define the function

$$
\varphi_{1}(t)=\int_{\mathbb{R}}|x|^{p+1} \varphi(x, t x) d x .
$$

For every integer $n \geq p+3$, there exists a constant $k_{n}$ such that $|\varphi(x, y)| \leq$ $k_{n}\left(1+\left(x^{2}+y^{2}\right)^{1 / 2}\right)^{-n}$ for all $x, y \in \mathbb{R}$. Therefore, $\varphi_{1}$ is a continuous function on $\mathbb{R}$ satisfying

$$
\left|\varphi_{1}(t)\right| \leq k_{n} \int_{\mathbb{R}} \frac{|x|^{p+1} d x}{\left(1+\left(x^{2}+t^{2} x^{2}\right)^{1 / 2}\right)^{n}} \leq k\left(1+t^{2}\right)^{-1-p / 2}
$$

for some $k>0$. In particular, $\varphi_{1} \in L_{1}(\mathbb{R})$.

As we mentioned, for each function $\psi \in \mathscr{D}(\mathbb{R})$ there exists an even function $\varphi \in S\left(\mathbb{R}^{2}\right)$ with $\hat{\varphi} \in \mathscr{D}(G)$ satisfying $\psi(\xi)=\int_{\mathbb{R}^{2}}|x \xi-y|^{p} \varphi(x, y) d x d y$ $=\int_{\mathbb{R}}|\xi-t|^{p} \varphi_{1}(t) d t=\left(|t|^{p} * \varphi_{1}\right)(\xi)$. We can use Lemma 1 from [10] to verify 
that $\hat{\psi}(t)=C_{p}|t|^{-1-p} \hat{\varphi}_{1}(t)$ for all $t \in \mathbb{R}, t \neq 0$. Hence $C_{p} \varphi_{1}=\left(|t|^{p+1} \hat{\psi}(t)\right)^{\nu}=$ $\psi^{(p+1)}$ is the $(p+1)$ th fractional derivative of the function $\psi(\vee$ denotes the inverse Fourier transform).

Now we define the $(p+1)$ th fractional derivative of the function $\left\|e_{1}+t e_{2}\right\|^{p}$. For every $\psi \in \mathscr{D}(\mathbb{R})$, we put

$$
\left\langle\left(\left\|e_{1}+t e_{2}\right\|^{p}\right)^{(p+1)}, \psi\right\rangle=\int_{\mathbb{R}}\left\|e_{1}+t e_{2}\right\|^{p} \psi^{(p+1)}(t) d t .
$$

(The integral on the right-hand side exists by (9).) If the Fourier transform $\left(\left\|e_{1}+t e_{2}\right\|^{p}\right)^{-}$is regular, then the fractional derivative can be computed:

$$
\left(\left\|e_{1}+t e_{2}\right\|^{p}\right)^{(p+1)}=\left(|x|^{p+1}\left(\left\|e_{1}+t e_{2}\right\|^{p}\right)^{\wedge}(x)\right)^{\swarrow} .
$$

Theorem 6. If $p>0, p \neq 2,4,6, \ldots$, then (1) an arbitrary two-dimensional space $E$ admits the Levy representation with exponent $p$ and distribution $\gamma=$ $\left(1 / C_{p}\right)\left(\left\|e_{1}+t e_{2}\right\|^{p}\right)^{(p+1)}$ and (2) an exponent $p$ is exceptional for $E$ iff $\left(\left\|e_{1}+t e_{2}\right\|^{p}\right)^{(p+1)}=0$ on some open subset of $\mathbb{R}$.

Proof. For every function $\varphi \in S\left(\mathbb{R}^{2}\right)$ with $\hat{\varphi} \in \mathscr{D}(G)$ we have

$$
\begin{aligned}
\left\langle\left(\| e_{1}\right.\right. & \left.\left.+t e_{2} \|^{p}\right)^{(p+1)}(\xi), \int_{\mathbb{R}^{2}}|x \xi-y|^{p} \varphi(x, y) d x d y\right\rangle \\
& =C_{p} \int_{\mathbb{R}}\left\|e_{1}+t e_{2}\right\|^{p} \varphi_{1}(t) d t \\
& =C_{p} \int_{\mathbb{R}}\left\|e_{1}+t e_{2}\right\|^{p} d t \int_{\mathbb{R}}|x|^{p+1} \varphi(x, t x) d x \\
& =C_{p} \int_{\mathbb{R}^{2}}\left\|x e_{1}+y e_{2}\right\|^{p} \varphi(x, y) d x d y .
\end{aligned}
$$

The second statement follows now from Lemma 4.

Corollary. The exponent $p=1$ is exceptional for a two-dimensional space $E$ iff this space is not strictly convex.

Indeed, if $p=1$, then the $(p+1)$ th fractional derivative coincides with the ordinary second derivative. So the exponent $p=1$ is exceptional for $E$ iff $\left\|e_{1}+t e_{2}\right\|^{\prime \prime}=0$ on some open segment in $\mathbb{R} ;$ i.e., $\left\|e_{1}+t e_{2}\right\|$ is a linear function on some open segment in $\mathbb{R}$, and so $E$ is not strictly convex.

\section{BIBLIOGRAPHY}

1. A. L. Al-Husaini, Potential operators and equimeasurability, Pacific J. Math. 76 (1978), 1-7.

2. J. Bretagnolle, D. Dacunha-Castelle, and J. L. Krivine, Lois stables et espaces $L_{p}$, Ann. Inst. H. Poincaré (B) 2 (1966), 231-259.

3. I. M. Gelfand and G. E. Shilov, Generalized functions and operations over them, Generalized Functions 1, Glav. Izdat. Fiz. Mat. Lit., Moscow, 1959. (Russian) 
4. I. M. Gelfand, M. I. Graev, and N. Ya. Vilenkin, Integral geometry and connected topics of representation theory, Generalized Functions 5, Glav. Izdat. Fiz. Mat. Lit., Moscow, 1962. (Russian)

5. E. A. Gorin and A. L. Koldobskii, On potentials, identifying measures in Banach spaces, Dokl. Acad. Nauk SSSR 285 (1985), 270-273; English transl. in Soviet Math. Dokl. 32 (1985), 659-663.

6. __ On potentials of measures in Banach spaces, Sibirskii Mat. J. 28 (1987), 65-80.

7. C. D. Hardin, Isometries on subspaces of $L_{p}$, Indiana Univ. Math. J. 30 (1981), 449-465.

8. M. Kanter, The $L_{p}$-norm of sums of translates of a function, Trans. Amer. Math. Soc. 179 (1973), 35-47.

9. A. L. Koldobskii, On isometric operators in $L_{p}\left(X ; \mathbb{R}^{n}\right)$, Functional Analysis (A. V. Strauss, ed.) Ul' yanovsk. Gos. Ped. Inst., Ul'yanovsk 12 (1979), 90-99. (Russian)

10. __ On isometric operators in vector-valued $L_{p}$-spaces, Zap. Naucn. Sem. Leningr. Otd. Mat. Inst. Steklov (LOMI) 107 (1982), 198-203. (Russian)

11. __ Uniqueness theorem for measures in $C(K)$ and its application in the theory of random processes, Zap. Nauchn. Sem. Leningr. Otd. Mat. Inst. Steklov (LOMI) 119 (1982), 144153; English transl. in J. Soviet Math. 27 (1984), 3095-3102.

12. _ Convolution metrics on spaces of measures and E-isometries, Zap. Nauchn. Sem. Leningr. Otd. Mat. Inst. Steklov (LOMI) 157 (1987), 151-156. (Russian)

13. N. S. Landkof, Foundations of modern potential theory, Nauka, Moscow, 1966; English transl. Springer-Verlag, 1972.

14. W. Linde, Moments of measures on Banach spaces, Math. Ann. 258 (1982), 277-287.

15. __ On Rudin's equimeasurability theorem for infinite dimensional Hilbert spaces, Indiana Univ. Math. J. 35 (1986), 235-243.

16. $\frac{}{626}$, Uniqueness theorems for measures in $L_{r}$ and $C_{0}(\Omega)$, Math. Ann. 274 (1986), 617626.

17. W. Lusky, Some consequences of Rudin's paper " $L_{p}$-isometries and equimeasurability," Indiana Univ. Math. J. 27 (1978), 859-866.

18. A. Neyman, Representation of $L_{p}$-norms and isometric embedding in $L_{p}$-spaces, Israel J. Math. 48 (1984), 129-138.

19. A. I. Plotkin, Continuation of $L_{p}$-isometries, Zap. Nauchn. Sem. Leningr. Otd. Mat. Inst. Steklov (LOMI) 22 (1971), 103-129.

20. __ An algebra, generated by translation operators, and $L_{p}$-norms, Functional Analysis, (A. V. Strauss, ed.), Ul' yanovsk. Gos. Ped. Inst., Ul' yanovsk 6 (1976), 112-121.

21. W. Rudin, $L_{p}$-isometries and equimeasurability, Indiana Univ. Math. J. 25 (1976), 215228.

22. K. Stephenson, Certain integral equalities, which imply equimeasurability of functions, Canad. J. Math. 29 (1977), 827-844.

23. V. M. Zolotarev, One-dimensional stable distributions, Nauka, Moscow, 1983. (Russian)

Leningrad Institute of Finance and Economics, Department of Mathematics, Ul. SADOVAYA 21, LENINGRAD, USSR (191023) 